\title{
QoS Supported IPTV Service Architecture over Hybrid-Tree-Based Explicit Routed Multicast Network
}

\author{
Chih-Chao Wen and Cheng-Shong Wu \\ Department of Electrical Engineering, National Chung Cheng University, Chia-Yi 62102, Taiwan \\ Correspondence should be addressed to Chih-Chao Wen, ccwen@ccu.edu.tw
}

Received 16 November 2011; Revised 14 March 2012; Accepted 30 March 2012

Academic Editor: János Tapolcai

Copyright (c) 2012 C.-C. Wen and C.-S. Wu. This is an open access article distributed under the Creative Commons Attribution License, which permits unrestricted use, distribution, and reproduction in any medium, provided the original work is properly cited.

\begin{abstract}
With the rapid advance in multimedia streaming and multicast transport technology, current IP multicast protocols, especially PIM-SM, become the major channel delivery mechanism for IPTV system over Internet. The goals for IPTV service are to provide two-way interactive services for viewers to select popular program channel with high quality for watching during fast channel surfing period. However, existing IP multicast protocol cannot meet above QoS requirements for IPTV applications between media server and subscribers. Therefore, we propose a cooperative scheme of hybrid-tree based on explicit routed multicast, called as HTERM to combine the advantages of shared tree and source tree for QoS-supported IPTV service. To increase network utilization, the constrained shortest path first (CSPF) routing algorithm is designed for construction of hybrid tree to deliver the high-quality video stream over watching channel and standard quality over surfing channel. Furthermore, the Resource Reservation ProtocolTraffic Engineering (RSVP-TE) is used as signaling mechanism to set up QoS path for multicast channel admission control. Our simulation results demonstrated that the proposed HT-ERM scheme outperforms other multicast QoS-based delivery scheme in terms of channel switching delay, resource utilization, and blocking ratio for IPTV service.
\end{abstract}

\section{Introduction}

As the rapid growth of broadband network applications with streaming transport over Internet, the Internet Protocol Television (IPTV) system has been widely deployed to provide multimedia service anywhere at any time. This is because IPTV enables digital service convergence of communications, computing, and media content over IP network with desired QoS guarantee [1]. From the perspective of the quality of experience (QoE), IPTV system operates as the same with broadcasting TV service, which would deliver the watching and surfing programs over different channels. However, the most difference is that IPTV works in a twoway interactive communications between service providers and subscribers. We need to consider the effective channel and delivery control problem to achieve video streaming with desired quality over Internet.

To efficiently satisfy multiple viewers' own quality requirements, IP multicast is considered a promising solution for IPTV application. Nevertheless, quality of service
(QoS) support to IPTV system still poses challenging issues for multicast channel delivery and resource utilization through IP networks. The QoS-supported IPTV multicast service architecture is to deploy an efficient multicast transmission system via IP multicast delivery tree with the integration of resource provisioning and channel admission control. The IP multicast delivery has the merit of efficient bandwidth saving; however, it is difficult to assign effective multicast channel to meet QoS requirements in consideration of multicast channel state labeling and channel switching delay [2]. Therefore, the original IP multicast is not designed for multimedia application to transport time-sensitive packet streaming with bandwidth reservation and QoS gurantee along point-to-multipoint (P2MP) multicast path for large amount of IPTV channel subscribers.

From the perspective of QoS requirement, IPTV channel change will impair the content quality of video streaming to speed up the surfing streams transmission. It usually depends on the group of picture (GOP) size between Intracoded frames (I-frames) in video stream sequence to determine 
the quality of watching channel and surfing channel. According to high quality video coding, a typical high definition (HD) video stream requires at least $10 \mathrm{Mbps}$ of I-frames transfer rate for IPTV watching channels, while a standard definition (SD) video stream requires 2-5 Mbps for lower quality video stream in IPTV surfing channels upon channel change [3].

From the perspective of network transmission performance, current IP multicast protocols can be enhanced by different multicast QoS routing mechanisms. The QoS-aware multicast routing protocol (QMRP) [4] was first to propose feasible multicast paths computation based on QoS metric for single path or multipath. Afterwards, the protocol independent multicast (PIM) protocol is based on receiver initiated routing decision to find the shortest path regardless of underlying unicast routing. For example, the typical PIM protocols are represented by source specific multicast (SSM) [5], and PIM-sparse mode (SM) [6]. Those two multicast protocols are integrated by $\mathrm{QoS}$ routing algorithm with traffic control to construct source tree, shared tree, and even hybrid-tree structures. Especially, this hybrid-tree multicast can be an alternative to improve IPTV QoS and achieve load balance of multicast traffic by combining advantages of above two multicast tree types: shared tree and source tree.

As the previous paper mentioned in [7], the hybridtree multicast is considered as a suitable solution for IPTV channel control and delivery to satisfy multicast QoS requirements. However, existing core functionality of PIM$\mathrm{SM}$ protocol in rendezvous point (RP) node still lacks the efficient control mechanism for hybrid-tree switchover operations to realize IPTV QoS multicast during channel change period. The reasons are explained as follows.

(i) High-level traffic control mechanism: RP router will aggregate all channel source streaming into the single shared tree until the link efficient bandwidth is overthreshold. The status report is a high-level control message detected by receivers. Therefore, the reaction time may be too slow to deal with unexpected QoS degradation and traffic congestion.

(ii) Two-pass switchover control operation: for channel change, multicast tree switchover is executed by RP node after receiver member-leave and rejoin request. By using two-pass switchover operation, RP node suspends the traffic aggregation from the specified source node; then channel traffic can be changed to new source node inefficiently.

Therefore, IPTV service provider must provide a costeffective multicast network control mechanism as an efficient channel delivery solution. In this paper, we propose the enhanced hybrid-tree-based multicast delivery scheme with explicit routed multicast, called as HT-ERM. To improve performance of QoS-supported IPTV multicast channel, our HT-ERM routing algorithm is designed based on constrained shortest path first (CSPF) [8], and HT-ERM channel admission control is employed by RSVP-TE mechanism [9]. In performance evaluation, the proposed HT-ERM scheme can improve IPTV delivery and channel control as compared with the other QoS multicast schemes.
The rest of this paper is organized as follows. We summarize past works of multicast QoS for IPTV in Section 2. In Section 3, we made assumptions of hybrid-tree multicast in related IPTV models. The Section 4 describes the HTERM control algorithm and multicast channel operations. Section 5 presents simulation results for IPTV service, and in Section 6 we give conclusions.

\section{Related Works}

QoS-supported IPTV services need to consider QoS guarantee, which involves with multicast delivery through core network, and channel selective control in user access network. Most of researches focus IPTV multicast QoS on two crucial subjects: multicast network resource control for IPTV watching channels and multiple surfing channel change delay control.

2.1. IPTV Multicast Channel Delivery and Change. The analysis of IPTV channel control for content delivery and channel change depends on those factors such as command processing time, network transmission delay time, streaming switchover delay time, and video-decoding time [10-12]. The most important key factors for channel control are affected by content transmission and streaming switchover through networks. In [11], the authors proposed multicast proxy IGMP scheme for channel prejoining to the expected IPTV channels by bulk delivery the popular watching channels and other subscribers can filter watching channel and switchover surfing channels in the same local network.

2.2. IPTV Multicast Network Resource Control. Many QoS multicast routing algorithms are proposed to compute the feasible multicast tree, so that can reduce traffic transmission delay and achieve efficient resource utilization. In traditional IP multicast network, the multicast routing algorithm lacks QoS control for network P2MP connections and traffic load balancing. Recently, QoS-aware multicast approaches, such as ECMP [13] and QMRP [4], are developed to solve scalability and resource allocation when a large amount of different multicast streams transmit to heterogeneous receivers through Internet.

The modified multicast equal-cost multipath (ECMP) scheme [13] has been applied in shared tree to enhance PIMSM or SSM-related IP multicast protocols. To achieve traffic balancing, multiple paths with equal cost are constructed to split the traffic from RP-shared tree. The centralized multicast traffic control approach is usually utilized by RP node to aggregate multicast traffic into the shared tree for IPTV watching channels and adjacent surfing channels together.

The QMRP is a well-known QoS-aware multicast routing control approach [4], which can compute optimal tree-path in single-path or multi-path mode to join multiast tree for IPTV channel delivery. When the traffic load on multicast tree link is over threshold, the multipath traffic distribution will be activated to diverse the traffic load, and thus switchover to the specific multicast path. 


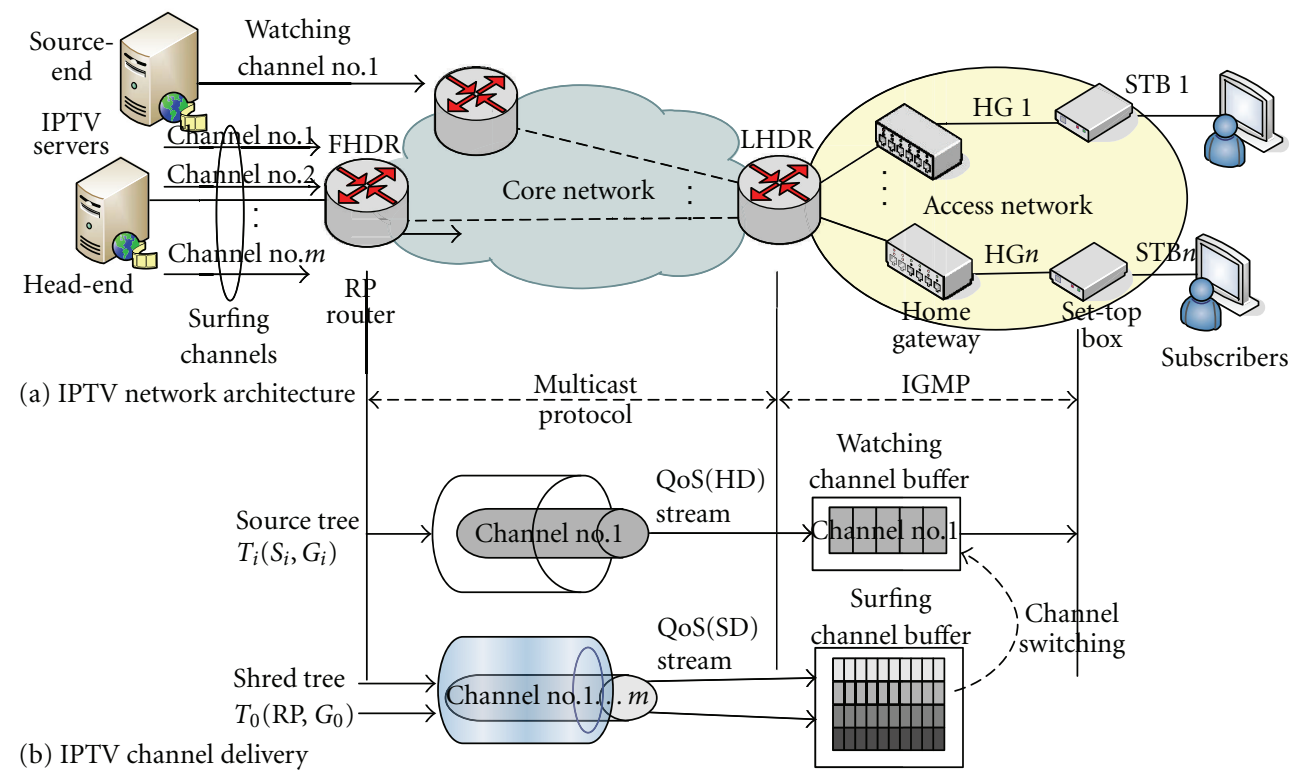

FIGURE 1: IPTV multicast network architecture consists of (a) core network and access network; IPTV channel delivered by (b) hybrid-tree based channel distribution scheme.

Furthermore, the proposed HT-ERM algorithm is to improve the overall IPTV channel delivery with efficient network resource utilization and fast channel change. The hybrid-tree-based multicast HT-ERM protocol integrates with hybrid multicast advantages to enhance functionality of current IP multicast protocol for IPTV QoS channel delivery control.

\section{IPTV System Model}

We use the IPTV system model to simplify the complicated service interaction between application level and network level. The IPTV channel quality states are assumed to abstract the channel dynamic behavior so as to introduce the proposed HT-ERM scheme in following sections.

3.1. Network Architecture for IPTV Service. The IPTV network architecture consists of multicast core network for channel distribution and local network for channel access as shown in Figure 1(a). In core network, IPTV channels are distributed and delivered by multicast protocol from firsthop designated router (FHDR) to last-hop designated router (LHDR). In local access network, the subscribers with terminal devices access IPTV channels via set-top box (STB) through home gateway (HG). For IPTV media service provider, the head-end (HE) server is to aggregate different basic quality channels for viewers' channel surfing and change behavior. The source-end server is to supply high quality program stream as a unique watching channel.
We model the IPTV system over IP multicast network. Assume that IPTV media servers can provide IPTV channels with source streams denoted by $S_{i}=\left\{S_{1}, \ldots, S_{m}\right\}$, and the subscriber members $M_{i}=\left\{M_{1}, \ldots, M_{n}\right\}$ can join any watching or surfing channel from its attached LHDR node $R_{i}$ to form the channel group $G_{i}=\left\{R_{1}, \ldots, R_{n}\right\}$. The collection of IPTV channels are delivered through multipoint-to-multipoint (MP2MP) connections $\left(S_{i}, G_{i}\right)$ between FHDR and LHDR over core network.

3.2. State Parameters for IPTV QoS Channel. In Figure 1(b), IPTV multicast channels are distributed between FHDR and LHDR. The source tree $T_{i}$ links carry the multiple high quality streams from their specific sources to the corresponding group. Each watching stream is transferred from watching channel buffer to STB. The surfing channel is aggregated by surfing streams over RP-based shared tree $T_{0}$ links with low quality. Each surfing channel is extracted from surfing channel buffer for fast channel switching during channel change.

The QoS-supported IPTV channel state is defined by two types of video quality: high definition (HD) and standard definition (SD). The HD video stream is paid per channel for high quality watching program, and SD video stream is normal quality used for fast channel surfing and free watching. Accordingly, the channel stream with higher QoS level is assigned to source-based multicast channel, and lower QoS level stream is delivered by shared multicast channel.

For QoS-supported IPTV channel state, the QoS level with HD is in steady-state function, and SD is in dynamic 


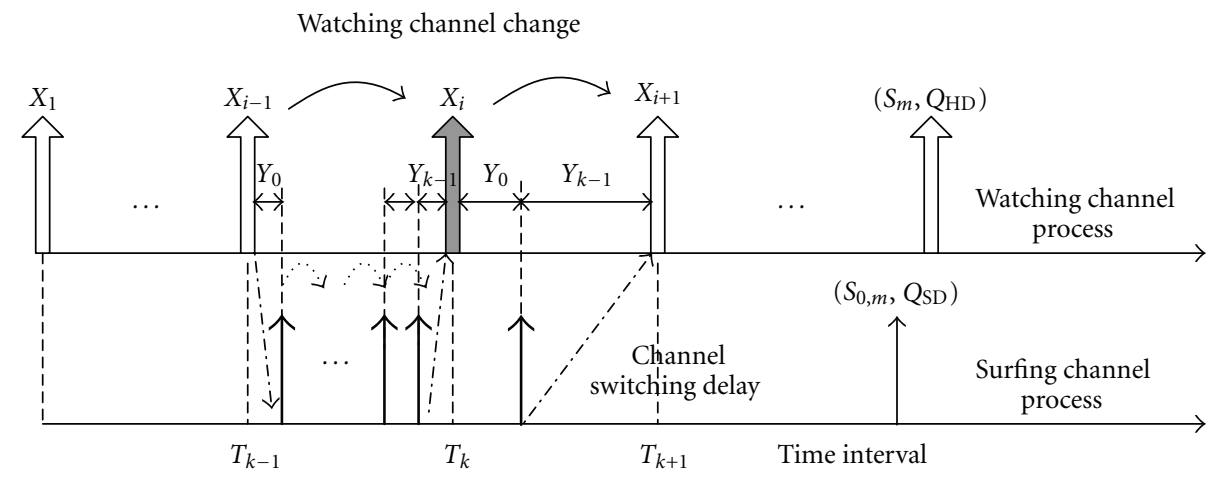

FIGURE 2: IPTV channel change process between watching and surfing channel state.

state. The IPTV QoS channel state can be represented by multicast tree symbol with QoS level $T(S, G, Q)$, which is characterized by

$$
T(S, G, Q)= \begin{cases}T_{\text {watch }}\left(S_{i}, G_{i}, Q_{q}\right), & i=1, \ldots, m ; q=\text { hd } \\ T_{\text {surf }}\left(S_{i, j}, G_{0}, Q_{q}\right), & i=0 ; j=1, \ldots, m, \\ & q=\mathrm{sd},\end{cases}
$$

where the parameter $S$ denotes the source nodes of IPTV channel, $G$ denotes the group identification for receiver joining, and $Q$ denotes the required stream QoS level with $\mathrm{HD} / \mathrm{SD}$. The $G_{i}$ is the multicast group joining to the specified source $S_{i}$ for watching channel no. $i$, and $G_{0}$ is the shared multicast group joining to the common source $S_{i, j}$ with RP router for aggregated surfing channels. In other words, the watching channel set is delivered by source tree $T_{i}\left(S_{i}, G_{i}, Q_{\mathrm{hd}}\right)$ for HD quality stream. The surfing channel is delivered by RP shared tree $T_{0}\left(S_{0, j}, G_{0}, Q_{\text {sd }}\right)$ for SD quality stream.

3.3. IPTV Channel Change Behavior. According to the definition of channel state, IPTV QoS channel can be obtained by watching state $T_{\text {watch }}$ and channel surfing state $T_{\text {surf }}$ in channel change process. Assume that viewers usually stay in watching channel $X_{i}$ with the state parameters $\left(S_{i}, Q_{\mathrm{HD}}\right)$, and the last surfing channel state with the state parameters $\left(S_{0, m}, Q_{\text {SD }}\right)$ will stop before next watching channel.

As the viewer making multiple channel changes, the random behavior of channel changing in surfing state can be modeled by a terminating renewal process [3]. Once channel change is occurred, the surfing state is zapping between surfing channels in transition state with random time interval $Y_{i}, i=0,1,2, \ldots$. channel switching process as shown in Figure 2. When channel change is stopped, we observed that the events $X_{i}$ of watching channel state always stay in steady state within the time interval $\left[T_{k}, T_{k+1}\right]$, where $T_{k}=$ $Y_{0}+Y_{1}+\cdots+Y_{k-1}$.

As IPTV channel change is a random process, the channel state may occur either in watching states or surfing states at any given time. We can figure out the joint channel state probability density function by

$$
P_{T}=p(k, q),
$$

where index $k$ is the channel number depending on program popularity, and index $q$ is the QoS stream quality distributed ratio over core network.

The channel popularity is the preference to the desired watching channel for most of viewers. According to Zipf's law [2], the probability $Z_{k}$ that a viewer will choose the $k$ th most popular channel is given by

$$
Z_{k}=\frac{c}{k^{s}}
$$

where $c$ is a constant to make the probabilities sum to 1 , and exponent $s$ is set to 1 . The selection of watching channel is first determined by Zipf's law. Then, the watching channel changes are occurred by poison process and terminated at next watching channel from surfing channel selection.

\section{The HT-ERM Scheme for IPTV Channel Delivery}

The proposed HT-ERM scheme has efficient hybrid-tree multicast operation for IPTV channel control and delivery as compared with the function of PIM-SM protocol.

4.1. HT-ERM Protocol Design. As shown in flow chart of Figure 3, we design the HT-ERM algorithm for multicast IPTV channels, including the hybrid-tree initialization, shared tree aggregation, source tree switchover and multicast admission control in following subsections.

4.1.1. Channel Initialization and Join. The RP node is initialized by IPTV channel aggregation for surfing channel joining requests in random process before watching channel selection. In additions, the initial parameters setting are shared tree $T_{0}$, shared group $G_{0}$, channel quality $Q$, and resource allocation threshold $\mathrm{BW}_{\mathrm{th}}$.

While receiving the channel request message, RP node checks the control message types (Join or Switchover) by source node ( $r p$ or $s$ ) and group member ( $g 0$ or $g i)$. The RP node is channel concentrator to update the surfing channel states through the shared tree $T_{0}$. The explicit routes of shared tree $T_{0}$ can be derived by computing CSPF (constrain 


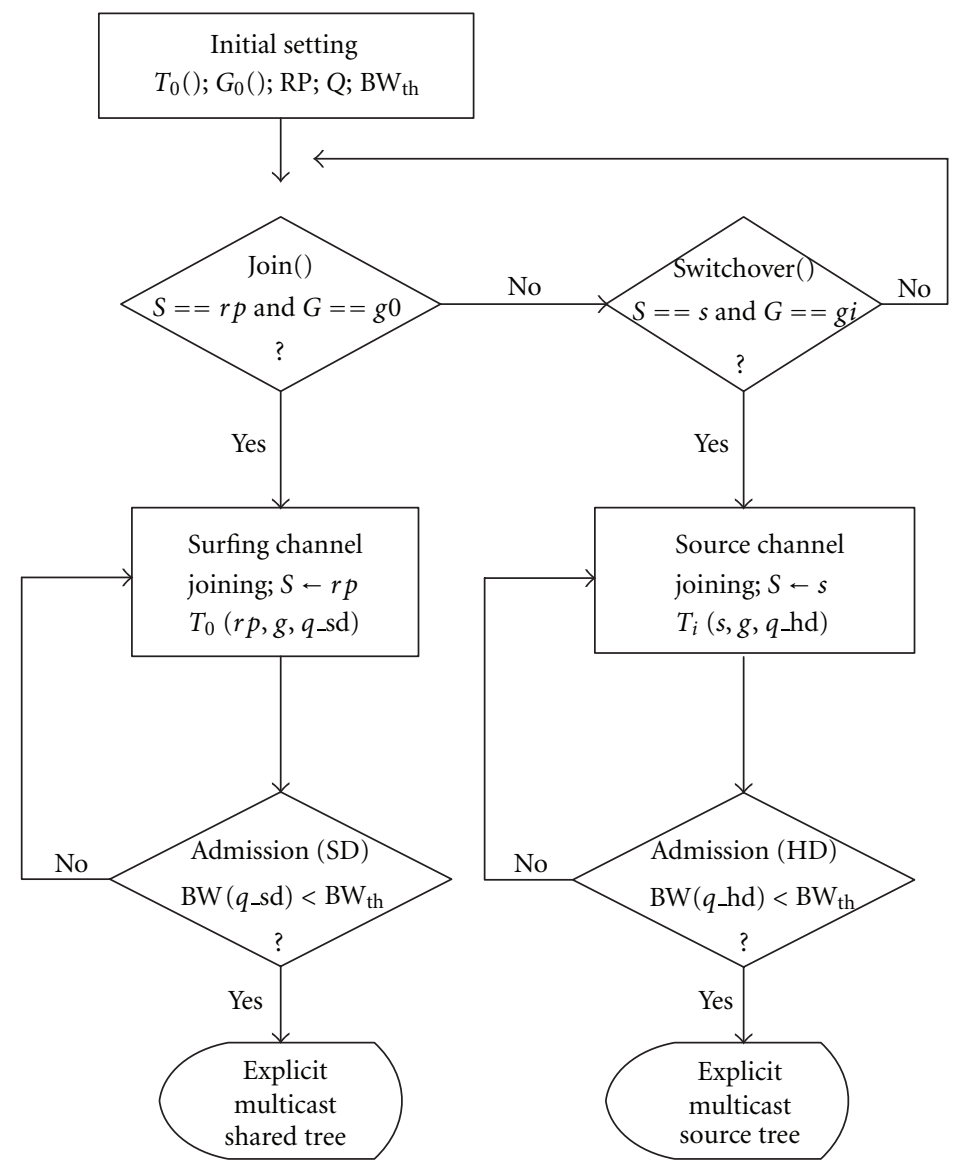

FIGURE 3: IPTV channel control algorithm for RP node.

shortest path first). The group source mapping table is generated. The surfing channels are expressed by explicit routed states in core routers as the multicast branching toward members. The more different IPTV channels are joined, the more network resource is utilized by multicast packet delivery.

4.1.2. Channel Change by Hybrid-Tree Switchover. As mentioned in Section 2, the channel change is a random process for viewer's behavior to select preferable watching channel. To reduce IPTV channel change time, the switchover control message is fast operated between RP shared tree and source tree simultaneously. The fast switchover mechanism, which is considered by link bandwidth utility and channel change time, can insert temporary SD quality stream from the same RP-aggregated shared tree as soon as possible to HD quality stream buffer for watching channel. After the new source tree switchover is finished, the HD quality media stream is delivered by specific source tree.

When the channel change request is occurred, the RP node executes switchover process for group members. After being informed by switchover control message, the source node computes the explicit routes for source tree $T_{i}$ by CSPF and then updates the channel states for watching stream delivery.
4.1.3. Channel Setup with Multicast Admission Control. According to the group member joining request with QoS requirements, the HT-ERM admission control will check the link bandwidth status for multicast hybrid tree. To guarantee QoS for multicast stream delivery, the upper bound of link utilization is defined by bandwidth threshold $\left(\mathrm{BW}_{\text {th }}\right)$ for efficient resource allocation that IPTV channel can carry the media streams through specific source tree $T_{i}$ and shared tree $T_{0}$. The bandwidth threshold $\left(\mathrm{BW}_{\mathrm{th}}\right)$ is to maximize the bandwidth usage for the total watching channel demands and additional channel change bandwidth estimation for network links.

Because the RSVP-TE is explicit routed signaling protocol, the admission control is used to reserve resource for hybrid-tree-based multicast. The surfing channel joining and watching channel switchover are admitted by comparing between available resource and bandwidth threshold $\left(\mathrm{BW}_{\mathrm{th}}\right)$. When the total bandwidth of $T_{i}$ and $T_{0}$ exceed the threshold $\mathrm{BW}_{\mathrm{th}}$, the blocking ratio for joining requests will be increasing due to detection of bandwidth overthreshold.

4.2. Channel Delivery Operational Differences between HTERM and PIM-SM. The differences between proposed HTERM and PIM-SM multicast protocol for channel delivery are listed as follows. 


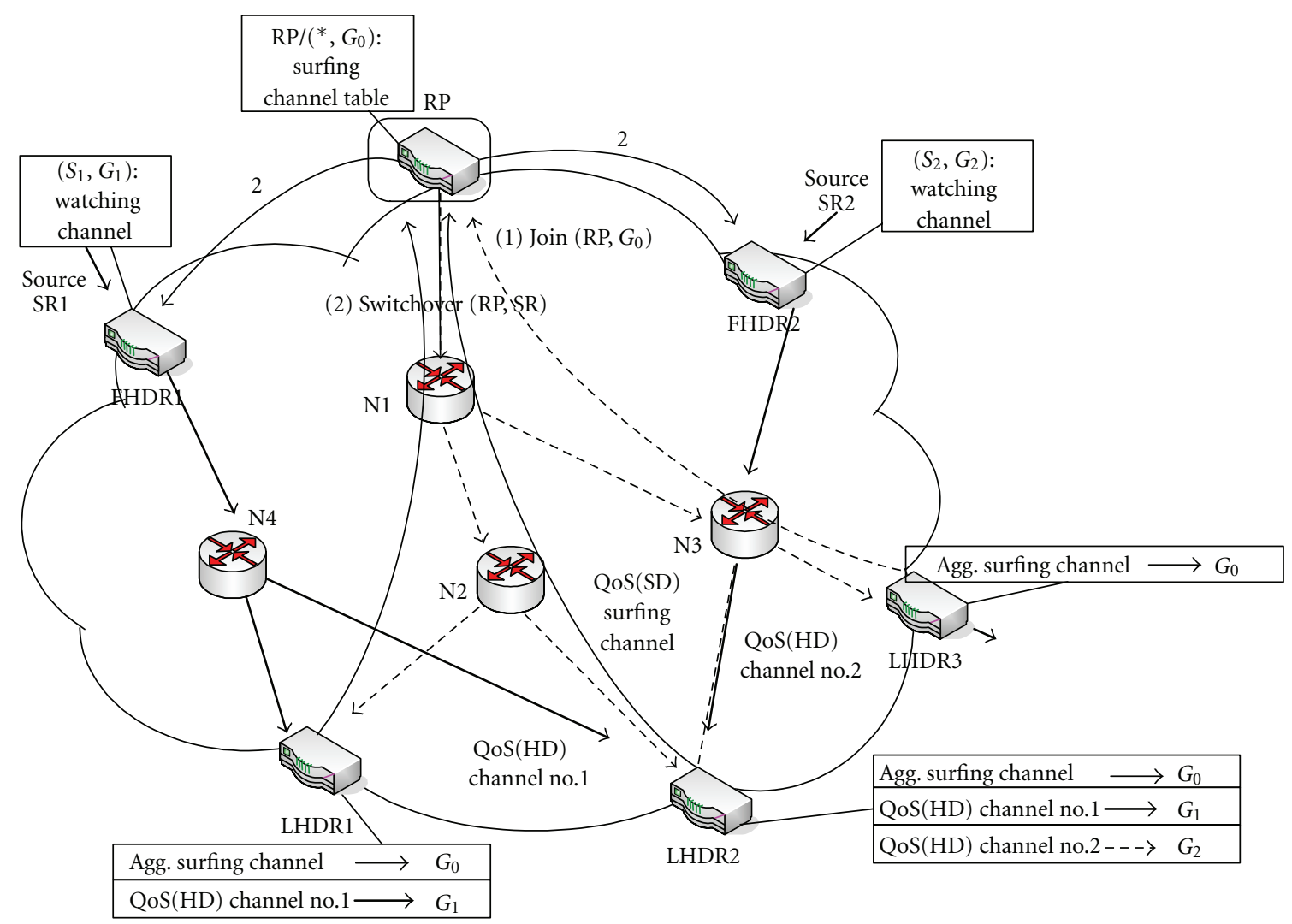

FIgURE 4: IPTV channel delivery through HT-ERM operations.

(1) Source group mapping table: because PIM-SM multicast routing is based on reverse path check, it is difficult to make accurate QoS estimation for bandwidth allocation from FHDR router of source node (or RP node) to multiple LHDR routers. The proposed HT-ERM scheme is based on mapping table to compute the source multicast explicit routed path forwarding toward all joining multicast group members.

(2) The modified control messages: Join/Leave (RP, $\left.G_{0}\right)$ and Switchover $\left(\mathrm{RP}, \mathrm{SR}_{i}\right)$ are adopted by the extended multicast protocol message of PIM-SM for IPTV channel setup and change. The control messages of HT-ERM are operated to enhance delivery performance between FHDR and LHDR.

To explain the HT-ERM protocol operations, we take an illustrative example in Figure 4. Firstly, the source node SR1 and SR2 will register to RP for surfing streams aggregation in RP shared tree. All group members distributed in LHDR nodes request RP to join to group $G_{0}$ for surfing channels. Secondly, upon receiving the join requests from LHDR 1-3, the RP function performs multicast channel initialization, and the group-source mapping table is updated by shared tree for surfing stream aggregation from SR1 and SR2. By information collecting from all sources and group members, the shared tree in hybrid multicast tree can be computed by CSPF for the explicit multicast routes of surfing channels.
In the scenario of LHDR1, the switchover message is completed to request new watching channel no. 1 from node SR1 via RP. However, in the scenario of LHDR2, not only one viewer requests watching the channel no. 1, one of views desires to change channel from the channel no. 1 to new channel no. 2. Instead of rejoining to SR2 for new channel no. 2 (S2, G2), RP can relay the control message Switchover (RP, SR) to inform old source node SR1 to retain the routes of subpath to LHDR2 for channel no. 1. The new source node SR2 is admitted to submit the media stream from channel no. 2 to LHDR2 by using the explicit routes with available bandwidth over (S2 and G2) source tree through core router N3. As for the scenario of LHDR3, the viewer is still in surfing state without decision making for specific watching channel.

\section{Performance Evaluation}

The performance measures of the proposed HT-ERM scheme for IPTV service are in terms of blocking ratio of admission control, resource utilization, and channel change delay over multicast core network and access network employed as performance. As compared with two underlying protocols, PIM-SM with ECMP algorithm and QMRP with QSPF algorithm, the HT-ERM with CSPF algorithm will be verified as a valid QoS supported IPTV multicast approach.

5.1. Simulation Parameters Setup. The simulation is conducted over two different network topologies. The first one 
is the random graph RandNet with 100-node and 294-link, generated by GT-ITM [14] network topology generator. The other is the fixed backbone graph NSFNet [15], which is abstracted from a real network model with 14 nodes and 42 bidirectional links. The multicast core network environment is setup by sources and receivers randomly attached to any network edge node as the designated router. In experiments, the number of join requests is measured from 500 to 5000 per 500 increasing step, to join different multicast channels. The group size is proportional to the total number of group member, and the joining requests from each node are uniformly distribution. From the aspect of viewer's random behavior, channel change joint state probability equals to the relationship with channel switching ratio $(\alpha)$ and channel popularity. The popular channels are usually assumed to stay within HD quality stream distribution ratio $(\beta)$. According to the empirical estimation by Zipf's law in (3), the cumulative probability of channel popularity is over $50 \%$ when $\beta$ is set to 0.2 (i.e., top 10 popular channels over total 50 channels). In our IPTV channel test scenario, those simulation parameters are summarized in Table 1.

5.2. Performance Metrics. In HT-ERM channel control simulation, the performance evaluations for hybrid-tree-based multicast scheme with different multicast routing algorithms are employed by PIM-like protocols. Table 2 lists hybrid multicast with three different multicast routing algorithms. The proposed HT-ERM scheme is used by CSPF algorithm. The conventional PIM-SM multicast scheme can be adopted by the equal cost multi-path (ECMP) algorithm. The QoSaware multicast routing protocol (QMRP) is used as PIMlike protocol based on QoS shortest path first (QSPF or QoS$\mathrm{SPF})$. The proposed HT-ERM scheme is source initial explicit routed multicast based on available bandwidth. The other schemes, multicast ECMP and multicast QSPF algorithms, are receiver-based multicast tree join by traversing the single path and/or multiple shortest path computations according to the link cost.

The performance metrics for multicast network delivery and QoS channel control are defined as following.

(i) Multicast Tree Setup Ratio. The multicast tree is computed by the specific on-tree node in RP node or source node. The tree setup ratio can be represented as the average number of multicast source and shared trees for multicast channel $(S, G)$ established by the joining requests from group members.

(ii) Multicast Forwarding Entries. The total number of multicast forwarding entries can be represented by the number of forwarding entries in multicast routing table per multicast router and the number of multicast router on multicast distribution tree $\mathrm{T}$ with group member $\mathrm{g}$. The number of multicast forwarding entries $\varepsilon$ is calculated by

$$
\varepsilon(T, g)=N_{e} \times N_{T}=\left(N_{s}+N_{b}+N_{t}+N_{l}\right) \times N_{T}
$$

where $N_{e}$ is the total number of forwarding entries in multicast routing table, that is, the total number of $(S, G)$ entries
TABLE 1: IPTV multicast simulation parameters.

\begin{tabular}{lc}
\hline IPTV channel delivery test conditions & Parameter \\
\hline Total number of IPTV channels & 50 \\
Channel switching ratio $\alpha$ & $0.25,0.5,0.8$ \\
HD QoS stream distribution ratio $\beta$ & 0.2 \\
HD/SD QoS stream bitrate & $10 / 2 \mathrm{Mbps}$ \\
Bandwidth threshold & $80 \%$ \\
Link bandwidth capacity & $500 \mathrm{Mbps}$ \\
\hline
\end{tabular}

TABLe 2: Hybrid multicast scheme with routing algorithms.

\begin{tabular}{lr}
\hline Hybrid-tree protocol & Multicast routing algorithm \\
\hline PIM-SM & Equal cost multipath (ECMP) \\
QMRP & QoS shortest path first (QSPF) \\
HT-ERM & Constrained shortest path first (CSPF) \\
\hline
\end{tabular}

in all multicast nodes for distribution tree $T$, including the root node number $N_{s}$, branching node number $N_{b}$, transit node number $N_{t}$, and leaf node number $N_{l}$. The number of multicast tree is denoted by $N_{T}$.

(iii) Maximum Multicast Resource Usage. The ratio of the utilized bandwidth is calculated in most traffic-congested link over multicast tree. The metric of resource usage $U$ is the total bandwidth consumption BW for QoS channels with HD and SD streams through multicast tree $T$ from joining requests of group member $g$ by

$$
U(Q, T)=\sum_{i \in T, q \in Q} \frac{\mathrm{BW}(i, q)}{C_{T}}
$$

where $C_{T}$ is the total tree link capacity over multicast network, and $\mathrm{BW}(i, q)$ is the reserved bandwidth for HD stream and SD stream over each link $i$ through distributed tree $T$.

(iv) Blocking Ratio. The ratio of rejection service request is divided by total requests under the admission control with bandwidth threshold $\mathrm{BW}_{\mathrm{th}}$. The metric of blocking ratio $\mathrm{B}$ is defined

$$
B(Q, G)=\sum_{g \in G} \frac{N_{R}}{N_{g}}
$$

where $N_{g}$ is the number of join requests from group member $G$ for available IPTV channels, and $N_{R}$ denotes the number of rejected member requests for specified QoS channel by admission control.

(v) Multicast Channel Change Delay. it is considered by channel processing delay, watching channel stream transmission, and surfing channel switching delay over multicast trees 
$T$ with group member size $g$. The metric of total switching delay time $\mathrm{D}$ can be summed up by

$$
\begin{aligned}
D(T, g)= & D_{1, \text { channel processing delay }} \\
& +D_{2, \text { surfing channel switching delay }} \\
& +D_{3, \text { watching channel transmission delay, }}
\end{aligned}
$$

where $D_{1}$ denotes channel processing delay, $D_{2}$ denotes surfing channel switching delay, and $D_{3}$ denotes watching channel transmission delay.

5.3. Comparisons of Multicast Network Delivery. We carried out following simulations over 100-node and 294-link random graph RanNet to evaluate the performance for multicast routing algorithms such as multicast ECMP, receiver-based QMRP- and RP-based HT-ERM scheme.

(1) Multicast Tree Setup Ratio Comparisons. The multicast tree setup ratio is measured by the average number of multicast source and shared trees computed by specific on-tree nodes per joining requests from group members. The main effect of multicast tree computation is determined by the number of active joining requests during channel change. The popular channel ratio $\beta$ with HD QoS stream distribution is set to 0.2 . With incremental channel switching ratio $\alpha$ by $0.25,0.5$, and 0.8 , we observed the setup trend of multicast tree for multicast channel $(S, G)$ computed by proposed HTERM routing schemes in RP and source nodes. Figure 5 shows the results for multicast tree setup ratio versus the average number of group member requests that compared HT-ERM scheme with CSPF, PIM-SM protocol with ECMP, and PIM-SSM protocol with QSPF. We found that the lowest setup ratio of multicast tree for ECMP scheme can afford for large amount of new channel change requests to diverse traffic flows into multiple links of shared tree. It means more multicast tree setup leads to more computation and delivery resource consumption. We also derive that multicast trees setup is almost identical to channel switching ratio in HTERM scheme with parameter $\alpha=0.25,0.5,0.8$. When the switching ratio is increasing to 0.8 , the multicast tree setup ratio of HT-ERM scheme still outperforms that of QMRP. That is because RP node uses HT-ERM with CSPF algorithm to gain the minimum hybrid-tree setup ratio with shared and source tree, instead QMRP is based on receiver multipath for source trees setup.

(2) Multicast Forwarding Entry Comparisons. Multicast forwarding entry is represented as the control overhead of multicast channel state maintenance in multicast forwarding table for network delivery through the multicast delivery tree. The total number of forwarding entries $(S, G)$ consists of root node number, branching node number, transit node number, and leaf node number over the distribution tree with group member. The increasing size of multicast forwarding entries is proportional to the number of group member joining requests because of grafted subpath from multicast branching node. Therefore, the performance for forwarding entry scalability may be affected by routing control scheme.

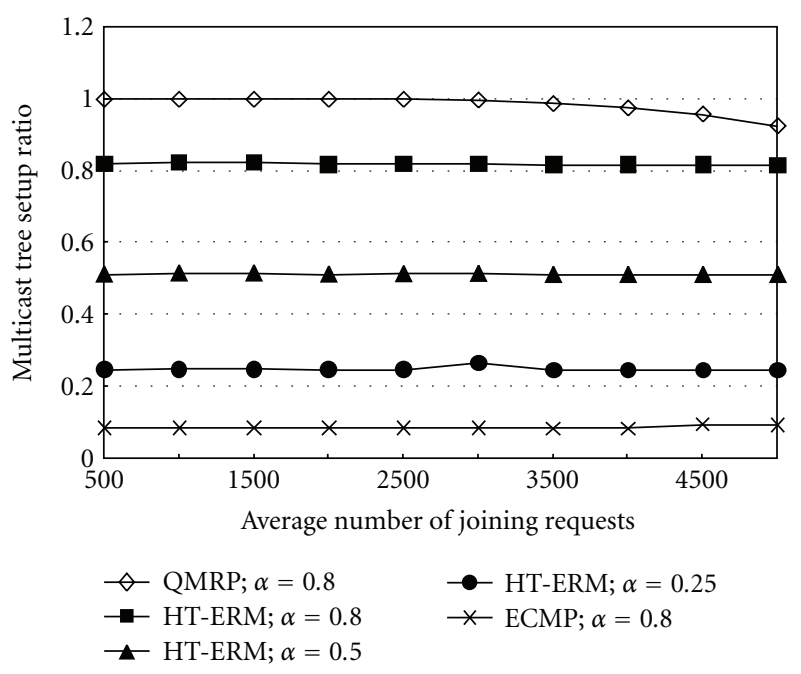

Figure 5: Multicast tree setup ratio versus group member joining requests.

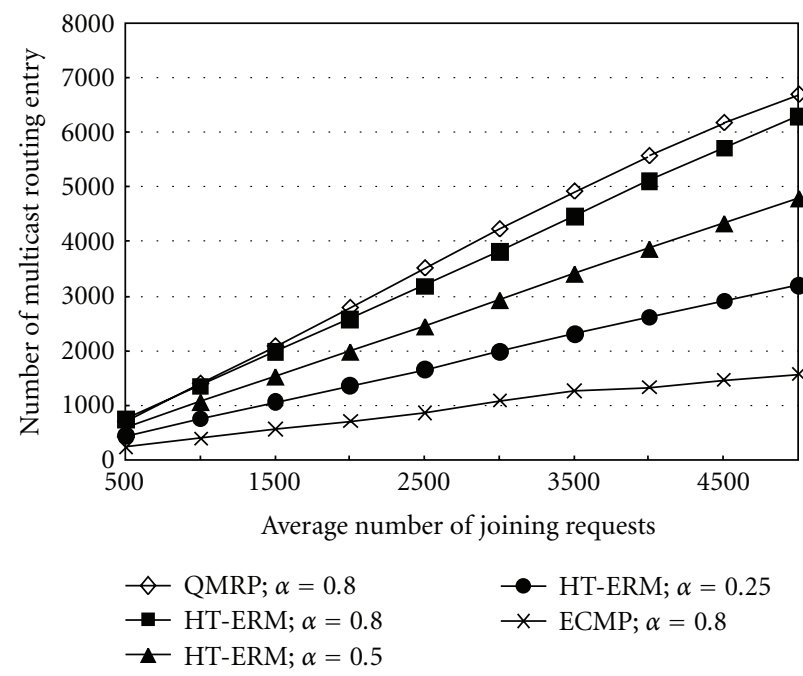

FIGURE 6: Comparisons of multicast forwarding entry size.

Figure 6 shows the comparison of ECMP, QMRP, and HT-ERM scheme with number of the forwarding entry number versus the average number of group joining requests. We found that the number of multicast forwarding entries for HT-ERM is increasing largely from 3,199 to 6,301 (i.e., the raising ratio is over $50 \%$ ) in condition of HD QoS stream distribution ratio $(\beta=0.2)$ and multicast channel switching ratio $(\alpha=0.8,0.5$ and 0.25$)$, when group joining member requests are more than 5000 times. When switching ratio is set to 0.8 , the growth of forwarding entry size computed by QMRP is a little greater than that of HT-ERM. In contrast, HT-ERM uses the explicit routes in RP-shared tree that can reduce more forwarding entries in multicast trees. However, ECMP is used to compute the unique shared tree, so that forwarding entry size is almost the same. 


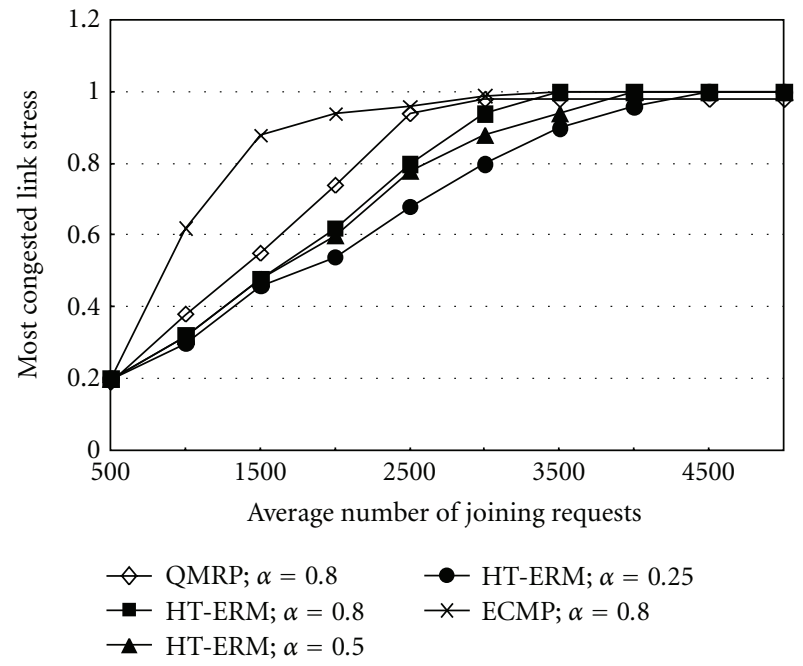

FIGURE 7: Multicast tree link resource usage versus group joining requests.

(3) Multicast Tree Link Resource Usage Comparisons. In multicast resource usage comparison, the multicast tree link stress is estimated by summation with the various channel switching ratio $(\alpha=0.8,0.5$, and 0.25$)$, under the HD QoS stream distribution ratio $(\beta=0.2)$. In Figure 7 , we observed that HT-ERM achieves the best resource efficiency utilized on the most congested multicast tree link as compared with ECMP and QMRP approach before link resource is overutilized by at the number of joining requests up to 4000 . In other words, the resource usage control of the proposed HT-ERM scheme is efficient by using CSPF algorithm because the explicated multicast routing can limit bandwidth threshold to redistribute heavy traffic loads between the shared tree and source trees over entire network topology.

(4) Joining Request Rejection Ratio Comparisons. The blocking for joining request is caused by insufficient resource allocation and high-level QoS request. The admission control can detect the available bandwidth in advance before accepting the joining requests with channel access. On the other hand, high-level QoS request may be rejected by either source node or RP node when multicast tree is switching over the specific links of source tree. By adjusting the various channel switching ratio $(\alpha=0.8,0.5$, and 0.25$)$ and HD QoS stream distribution ratio $(\beta=0.2,0.5)$, we evaluate the performance for large amount of joining requests for multicast tree setup by comparing multicast routing algorithm with ECMP, QMRP, and HT-ERM. We found that the rejection ratio of each routing algorithm is increasing by large amount of the joining requests from group members at higher channel switching ratio $(\alpha=0.8)$ and higher QoS stream distributed ratio $(\beta=0.5)$, as shown in Figure 8 . Especially, even in the worst conditions of channel switching ratio $(\alpha=0.8)$ and HD QoS distribution ratio $(\beta=0.5)$, the performance of rejection ratio for HT-ERM routing algorithm is better than that of ECMP and QMRP routing algorithm. As the result,

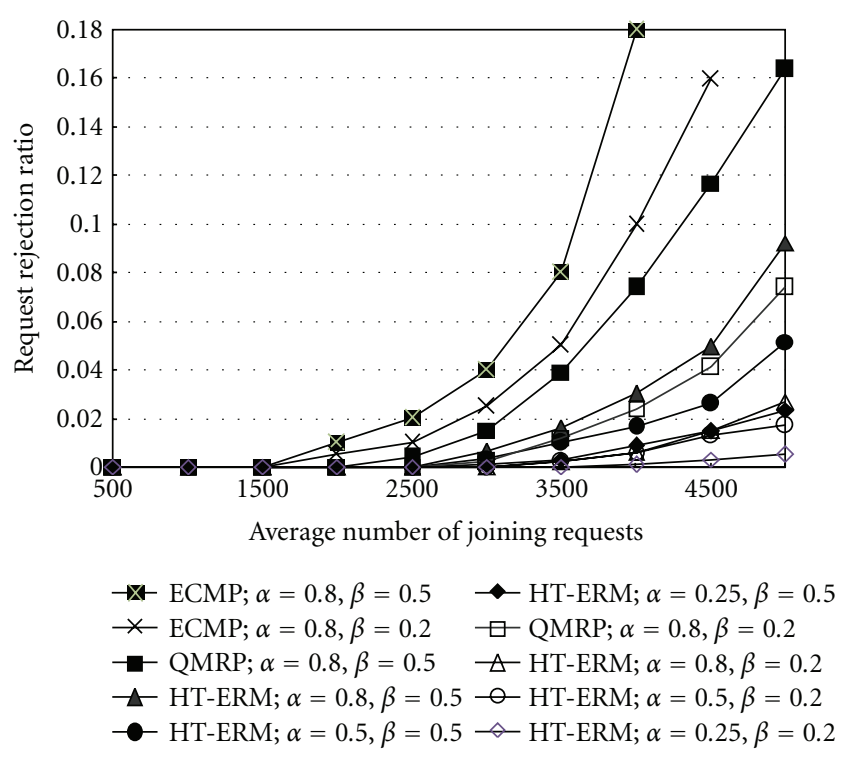

FIGURE 8: Request rejection ratio versus group joining requests.

we can prove that the CSPF algorithm of proposed HT-ERM can achieve efficient resource allocation and effective admission control for QoS requirements.

5.4. Comparisons of IPTV Channel Control. We carried out following simulations over real backbone network NSFNet with 14-node and 42-link to evaluate the performance for different IPTV multicast protocols PIM-SM, QMRP, and HT-ERM applied by associated channel control scheme.

(1) Channel Change Delay. In metric definition (7), the major channel delay effects are caused by surfing channel switching delay $D_{2}$, and watching channel transmission delay $D_{3}$. We simulated a large amount of groups to receive different IPTV watching channels over NSFNet topology and obtained the results of channel change delay. The Figure 9 demonstrates comparisons among different multicast tree construction for successful channel change from 10 to 50 at each access node.

The traditional RP-shared tree setup with ECMP algorithm leads to the largest transmission delay during channel change. The traffic of multipath is separated on the shared tree links so as to result in the large switching control delay; however, the multi-path can reduce the traffic load. As the QRMP approach constructs the source tree by QoS routing algorithm (i.e., QSPF), the IPTV channel traffic distribution can be diversified by different source tree links. The proposed HT-ERM can construct hybrid multicast tree based on CSPF algorithm to reduce the switching latency by source and shared tree switchover. As compared by QSPF routing algorithm, the proposed CSPF of HT-ERM scheme improves the performance for channel change delay. The importance of this simulation result indicates that the source-initiated QoS routing algorithm can achieve better channel switching 


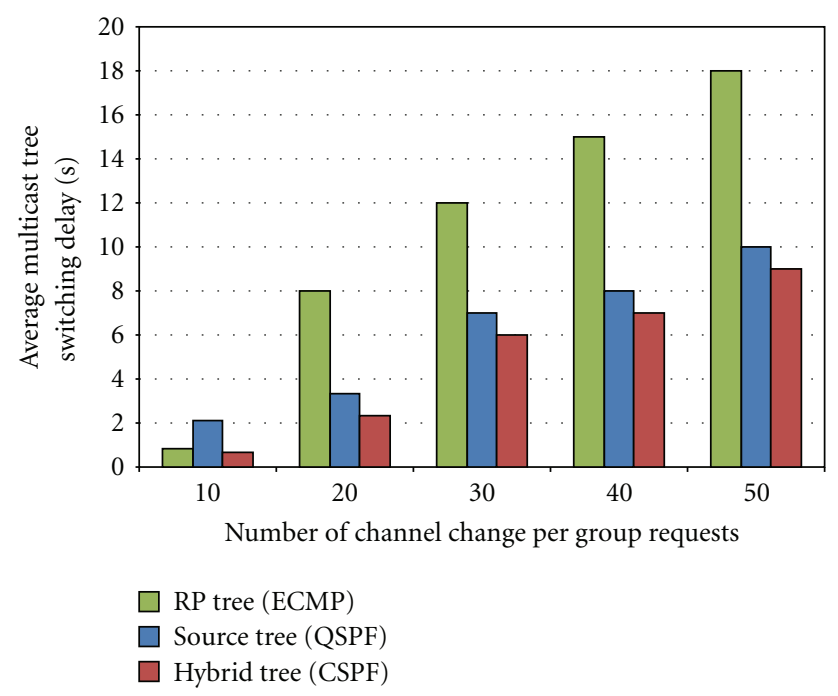

FIGURE 9: Channel change delay comparisons by multicast tree switching and transmission.

control performance than that of the receiver-initiated routing algorithm. Therefore, the proposed HT-ERM scheme can reduce the channel change delay by enhancing the hybridtree operation with efficient switchover mechanism for RPcentralized control.

(2) Channel Blocking Ratio. To simulate the blocking ratio for practical IPTV channel service, multicast routing protocols are employed by using different routing algorithm for number of IPTV channel request per group joining. By adjusting the channel switching ratios $(\alpha=0.25,0.5$, and 0.8 ) for different test scenarios, the HD QoS IPTV channels are requested to join per group member using PIM-SM with ECMP, QMRP with QSPF, and the proposed HT-ERM with CSPF. The results of Figure 10 indicate that the HT-ERM scheme with CSPF outperforms the other two QSPF and ECMP algorithm beneath the average switch ratio $(\alpha=0.5)$, while the number of channel joining requests is increasing from 10 to 50. We observe that the traditional PIM-SM multicast using ECMP resulted in the highest blocking ratio due to the traffic aggregation over shared tree with the same routing path, regardless of the multiple parallel links. In additions, note that the channel blocking ratio of HT-ERM is rising sharply by admission control at higher switching ratio $\alpha=0.8$.

(3) Channel Resource Usage. Figure 11 shows the results of resource usage for delivering watching streams over the requested IPTV channels using different multicast approaches. The resource consumptions are nearly even with those multicast approaches: PIM-SM (ECMP), QMRP (QSPF), and proposed HT-ERM (CSPF) at any channel switching ratio $(\alpha=0.8,0.5,0.25)$. For the effect of traffic load balancing, QMRP and HT-ERM scheme can achieve the better link usage performance as compared by PIM-SM while the number of channel joining is increasing to 50. Because

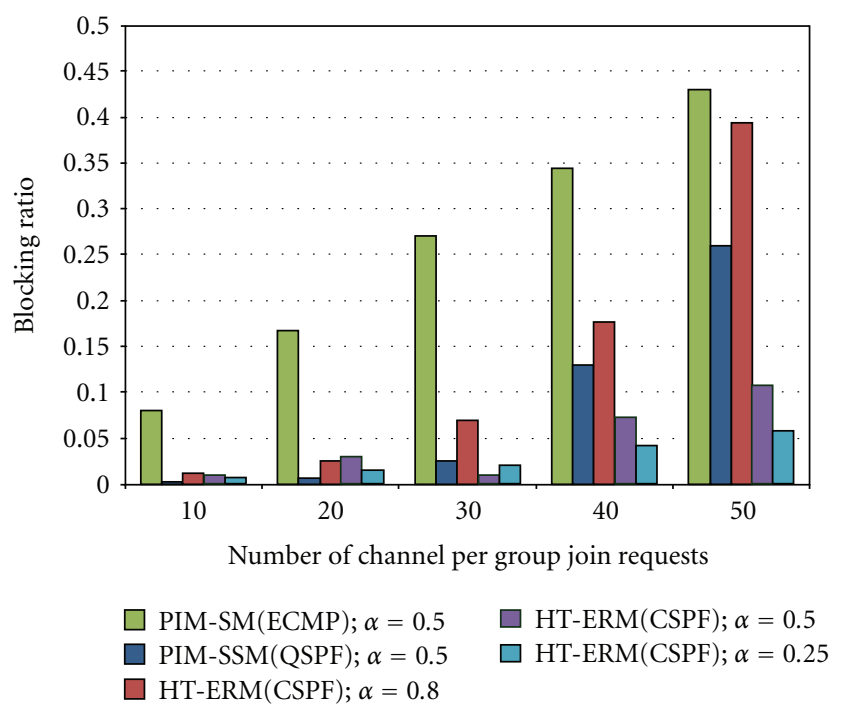

FIgURE 10: Comparisons of blocking ratio for channel control.

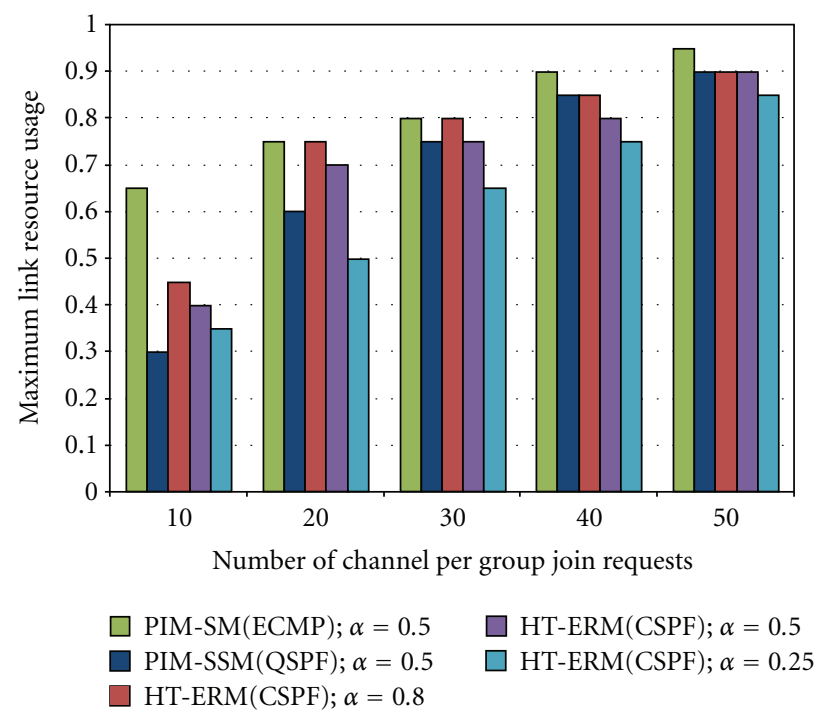

FIGURE 11: Comparisons of resource usage for IPTV channels.

the QSPF routing algorithm is based on receiver's multiple tree-nodes joining decision, the channel traffic distribution can perform better than that of the proposed HT-ERM at lower channel joining requests of 10 and 20. However, the maximum link utilization of HT-ERM performs nearly the same resource usage at higher channel joining requests more than 30 . This is the cross-effect caused by network congestion and traffic control. Therefore, the proposed HT-ERM scheme can operate hybrid-tree efficiently via enhanced RP functionality which makes better resource management for HD/SD channel stream utilization and traffic load distribution by hybrid-tree multicast channel service. 


\section{Conclusions}

As the killer application in future Internet, IPTV service needs to provide the effective and efficient operations for channel delivery and control. The hybrid-tree-based multicast IPTV has been validated to supply watching channels and surfing channel services. However, we need to overcome the drawbacks in RP functionality and switchover mechanism to achieve effectiveness and efficiency in hybrid-tree multicast channel control and delivery. To solve the QoS degradation problems of multicast network utilization and IPTV channel change delay, the hybrid-tree-based explicit routed multicast (HT-ERM) scheme is proposed to enhance current multicast protocols for QoS-supported IPTV service. The main contributions focus on performance improvement for multicast network delivery and IPTV channel control, including the reduction of maximum link resource utilization, fast switchover mechanism for channel change delay, and admission for multicast QoS channel setup. In networklayer level, HT-ERM provides flexible approach to design hybrid-tree-based IPTV multicast for IP multicast protocols. We also validate multicast solution to QoS-supported IPTV channel control and delivery by simulation. The result shows that the proposed HT-ERM outperforms existing QoS multicast approaches applied by PIM-related protocols while retaining QoS guarantee.

\section{References}

[1] Y. Xiao, X. Du, J. Zhang, F. Hu, and S. Guizani, "Internet protocol television (IPTV): the killer application for the nextgeneration internet," IEEE Communications Magazine, vol. 45, no. 11, pp. 126-134, 2007.

[2] W. Sun, X. Luo, K. Lin, and Y. Guan, "Performance analysis of a finite duration multichannel delivery method in IPTV," IEEE Transactions on Broadcasting, vol. 54, no. 3, pp. 419-429, 2008.

[3] D. E. Smith, "IP TV bandwidth demand: multicast and channel surfing," in Proceedings of the 26th IEEE International Conference on Computer Communications (INFOCOM '07), pp. 2546-2550, Anchorage, Alaska, USA, May 2007.

[4] S. Chen, K. Nahrstedt, and Y. Shavitt, "QoS-aware multicast routing protocol," IEEE Journal on Selected Areas in Communications, vol. 18, no. 12, pp. 2580-2592, 2000.

[5] H. Holbrook and B. Cain, "RFC 4607: Source-Specific Multicast for IP," Internet Engineering Task Force, August 2006.

[6] B. Fenner, M. Handley, H. Holbrook, and I. Kouvelas, "Protocol Independent Multicast-Sparse Mode (PIM-SM): Protocol Specification (Revised)," RFC 4601, August 2006.

[7] C. C. Wen, C. S. Wu, and M. T. Yang, "Hybrid tree based explicit routed multicast for QoS supported IPTV service," in Proceedings of the IEEE Global Telecommunications Conference (GLOBECOM '09), December 2009.

[8] M. Bag-Mohammadi, N. Yazdani, and S. Samadian-Barzoki, "On the efficiency of explicit multicast routing protocols," in Proceedings of the 10th IEEE Symposium on Computers and Communications (ISCC '05), pp. 679-685, June 2005.

[9] S. Yasukawa, M. Uga, H. Kojima, and K. Sugisono, "Extended RSVP-TE for Multicast LSP Tunnels," IETF draft, June 2003.

[10] C. Sasaki, A. Tagami, T. Hasegawa, and S. Ano, "Rapid channel zapping for IPTV broadcasting with additional multicast stream," in Proceedings of the IEEE International Conference on Communications (ICC '08), pp. 1760-1766, May 2008.

[11] Y. Kim, J. K. Park, H. J. Choi et al., "Reducing IPTV channel zapping time based on viewer's surfing behavior and preference," in Proceedings of the IEEE International Symposium on Broadband Multimedia Systems and Broadcasting (BMSB '08), April 2008.

[12] H. Joo, H. Song, D. B. Lee, and I. Lee, "An effective IPTV channel control algorithm considering channel zapping time and network utilization," IEEE Transactions on Broadcasting, vol. 54, no. 2, pp. 208-216, 2008.

[13] Cisco Technical Support Module, Load Splitting IP Multicast Traffic over ECMP, Cisco Systems, 2007.

[14] B. Chinoy and H.-W. Braun, "The national science foundation network,” Technical Report GA-A210029, SDSC, 1992.

[15] K. L. Calvert, E. W. Zegura, and S. Bhattacharjee, "How to model an internetwork," in Proceedings of the 1996 15th Annual Joint Conference of the IEEE Computer and Communications Societies (INFOCOM '96), pp. 594-602, March 1996. 

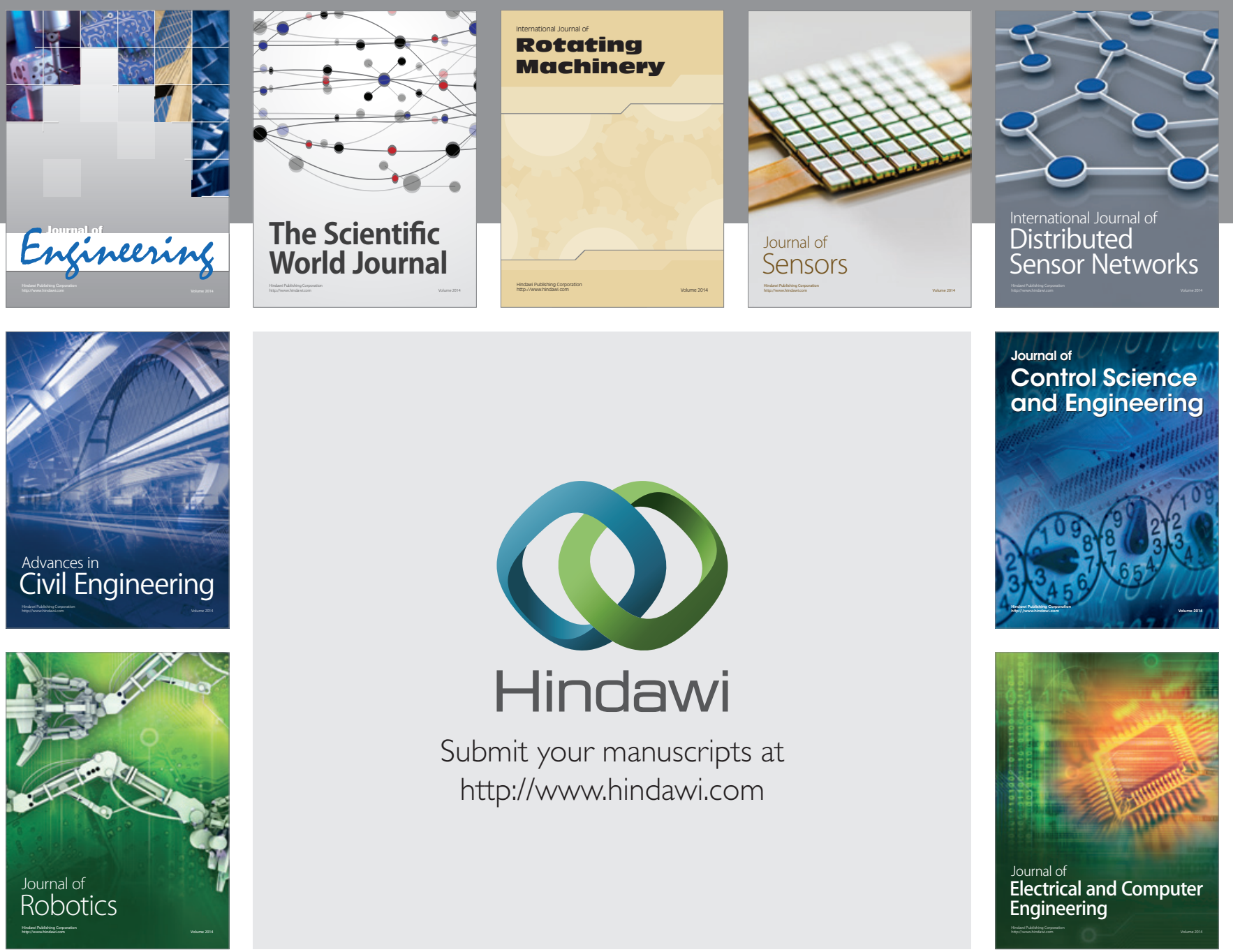

Submit your manuscripts at

http://www.hindawi.com
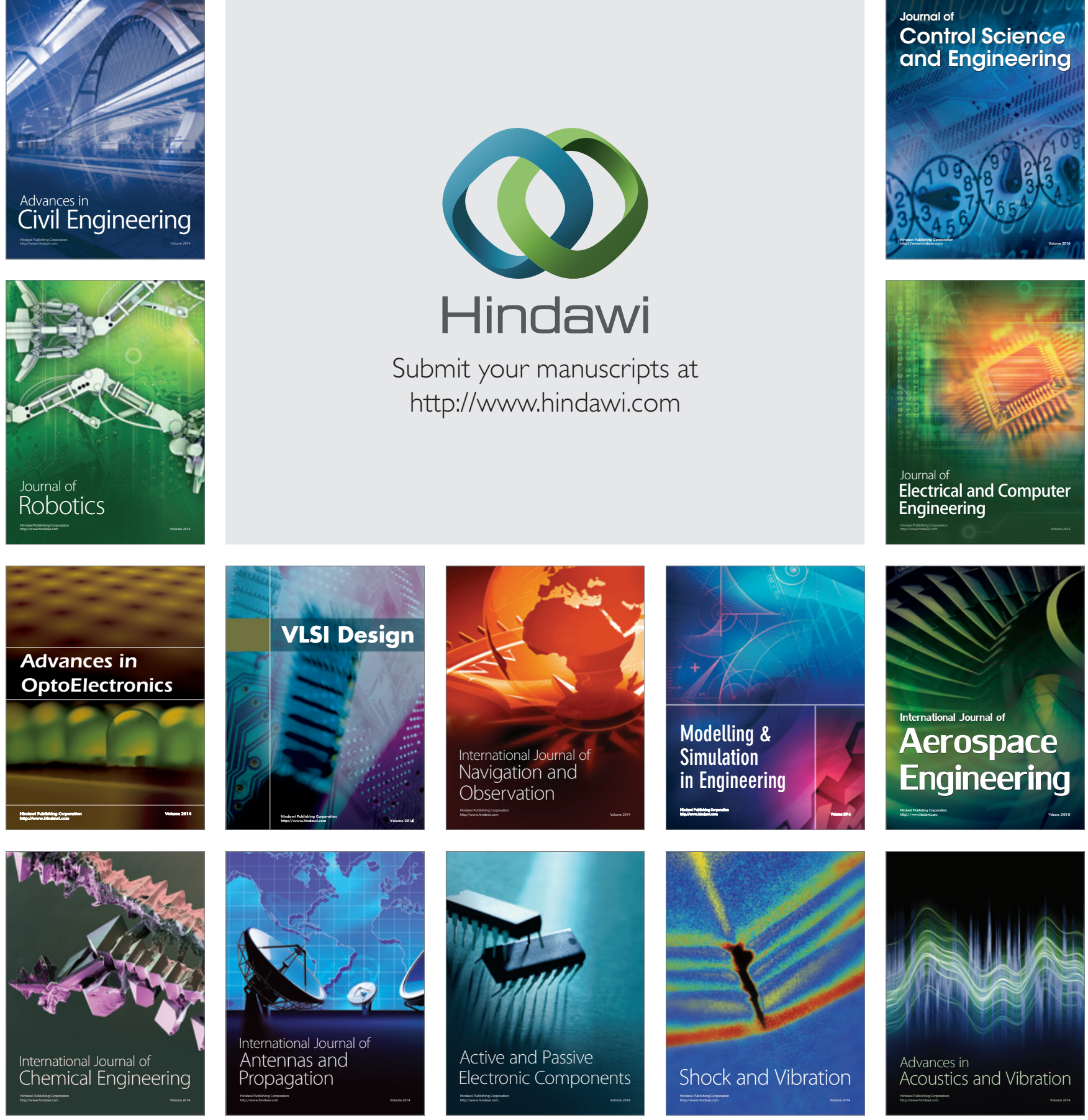